\title{
\begin{tabular}{l|l} 
Chapter 42 & Disorders of Haemostasis
\end{tabular}

\author{
S.J. Machin and F. Matthey
}

The normal haemostatic process is carefully balanced so that haemorrhage is promptly arrested and inappropriate thrombosis does not occur. There are numerous causes of acquired defects of haemostatic failure and these are often associated with multisystem disease, drug therapy or particular physiological events such as pregnancy, the newborn infant or old age. The initial problem is to clinically recognize a bleeding tendency and then to define the defect by specific laboratory tests before the appropriate prophylactic or therapeutic measures can be instigated.

A careful clinical history and physical examination should be undertaken and the initial difficulty is often to determine whether bleeding is due to a local factor, such as a peptic ulcer, or to an underlying haemostatic defect. In particular, continual oozing from venepuncture and drip sites, extensive petechiae and bruising at pressure areas or the sites of recent intramuscular injections and steady blood loss from postoperative drainage tubes are often signs of impending haemostatic failure. Paradoxically patchy skin cyanosis with the later development of superficial gangrene of the limbs or face may signify a consumptive coagulopathy before excessive clinical bleeding is apparent.

Initially a series of simple laboratory screening tests which are easy to perform and give reliable results quickly should be undertaken. A suggested widely available screening procedure is given in Table 42.1. The normal range for each test performed in the particular laboratory involved should be known before any results can be interpreted. If the screening tests suggest an abnormality further specialized investigations such as specific coagulant factor assays and immunological studies should be carried out to define precisely the defect and its severity. The technical details of these various tests will not be discussed as these are available in numerous other practical manuals [52]. The basic physiology of the normal haemostatic process and the investigation of haemostatic disorders have been previously summarized in Chapter 9 .

\section{Disseminated Intravascular Coagulation}

Disseminated intravascular coagulation (DIC) occurs due to inappropriate and excessive activation of the haemostatic process [81]. This is initially present in a compensated state when the process produces no clinical symptoms and can only be demonstrated by laboratory tests. In the subacute or chronic form there is a slow activation of the haemostatic system with spontaneous bruising rather than major clinical bleeding episodes. If the triggering factor is severe enough, the clinical syndrome of uncompensated acute DIC will result, with a generalized haemorrhagic state and/or endorgan failure following blockade of the microvasculature by thrombus formation.

The major clinical problem and presenting feature of acute DIC is bleeding. This may manifest itself as generalized bruising, especially over dependent areas and pressure sites, or there may be generalized oozing or even more profuse bleeding. In surgical practice this may occur during the operative period, especially in situations involving extracorporeal circulations, massive blood transfusions and complicated prolonged procedures. Usually, however, it 
Table 42.1. Laboratory screening test to detect a haemostatic defect

\begin{tabular}{l} 
1. Coagulation factors \\
Prothrombin time \\
Thrombin time \\
Activated partial thromboplastin time \\
Fibrinogen level \\
2. Platelets \\
Platelet count \\
Blood film inspection \\
Bleeding time \\
3. Fibrinolysis \\
Euglobulin lysis time \\
D-D dimers \\
\hline
\end{tabular}

presents in the immediate 24-h postoperative phase, often whilst the patient is in an intensive care unit. The bleeding occurs from venepuncture sites, around intravenous lines, from the surgical incision and in-dwelling drainage tubes, and may also be generalized throughout the gastrointestinal tract, from the oronasopharynx with pulmonary haemorrhage and from the urogenital tract. In obstetric units postpartum bleeding from the vagina may be particularly severe. In approximately 5\%-10\% of cases microthrombotic lesions are present initially as clinical features. This usually presents with gangrene of the fingers or toes. Fibrin thrombi are most commonly observed in the micro-arterioles of the renal glomeruli and may cause acute renal failure. A variety of thrombotic skin lesions including purpura fulminans, gangrene, acrocyanosis and haemorrhagic bullae may result from dermal capillary thrombosis. The involved vessels and surrounding tissue show necrosis and haemorrhage. Platelet-fibrin thrombi in the pulmonary vasculature may cause the acute respiratory distress syndrome or shock lung. Transient central nervous system disturbances are also common due to focal ischaemia.

\section{Pathogenesis of DIC}

The potential underlying triggering mechanisms are numerous but acute DIC may be initiated in three basic ways.

1. Stimulation of the coagulation cascade by the entry of tissue thromboplastins containing a high phospholipid concentration into the bloodstream [68]. This occurs following extensive tissue trauma during surgery, by dissemination of malignant tissue and during an acute intravascular haemolytic episode. (i.e. following an incompatible blood transfusion).

2. Severe endothelial injury of the vessel wall will cause activation of the coagulation cascade mainly by the intrinsic pathway and kallikreinkinin system. This occurs in patients with Gram-negative septicaemia due to released endotoxins, with virus diseases, following extensive burns and may be exacerbated by prolonged hypotension, hypoxia and acidosis.

3. Direct induction of platelet activation. This can occur primarily in septicaemic and viraemic states and by antigen-antibody complexes. Platelet activation will also result secondarily from vessel wall endothelial damage and following thrombin generation by the coagulation cascade.

The final consequence following activation of the coagulation cascade is fibrin formation. Fibrinogen is a glycoprotein with a molecular weight of 340000 . It consists of a dimer with two identical subunits. Each subunit has three polypeptide chains termed $\mathrm{A} \alpha$ (molecular weight 66500), B $\beta$ (molecular weight 52000) and $\gamma$ (molecular weight 46500). Thrombin cleaves sequentially fibrinopeptides $\mathrm{A}$ and $\mathrm{B}$ from the $A \alpha$ and $B \beta$ chains forming fibrin monomers. These monomers spontaneously polymerize to form the fibrin clot. Factor XIIIa then catalyses the formation of new peptide bonds between the chains of adjacent fibrin molecules, followed by cross-linking of the chains. This stabilizes and increases the tensile strength of the fibrin clot. Circulating plasminogen is incorporated into the fibrin clot. Excessive fibrin strands and aggregates of activated platelets will cause partial blockage initially of the arterial microcirculation. Fibrin-platelet plugs act rather like a fine-mesh sieve and passing red cells are traumatized, leading to distorted fragmented microcytic red cells and a resultant intravascular haemolytic process.

Following intravascular thrombosis there is a localized secondary activation of the fibrinolytic pathway in an attempt to limit thrombus formation and to begin the repair process by lysing the formed platelet-fibrin plug. Tissue-type plasminogen activator ( $\mathrm{tPA}$ ) is released locally from the vascular endothelium at sites of fibrin deposition and binds readily with plasminogen incorporated into the fibrin-platelet clot. Plasmin generated in the fibrin clot is protected from the inhibitory action of alpha ${ }_{2}$-antiplasmin. If an excessive generalized release of $\mathrm{tPA}$ 


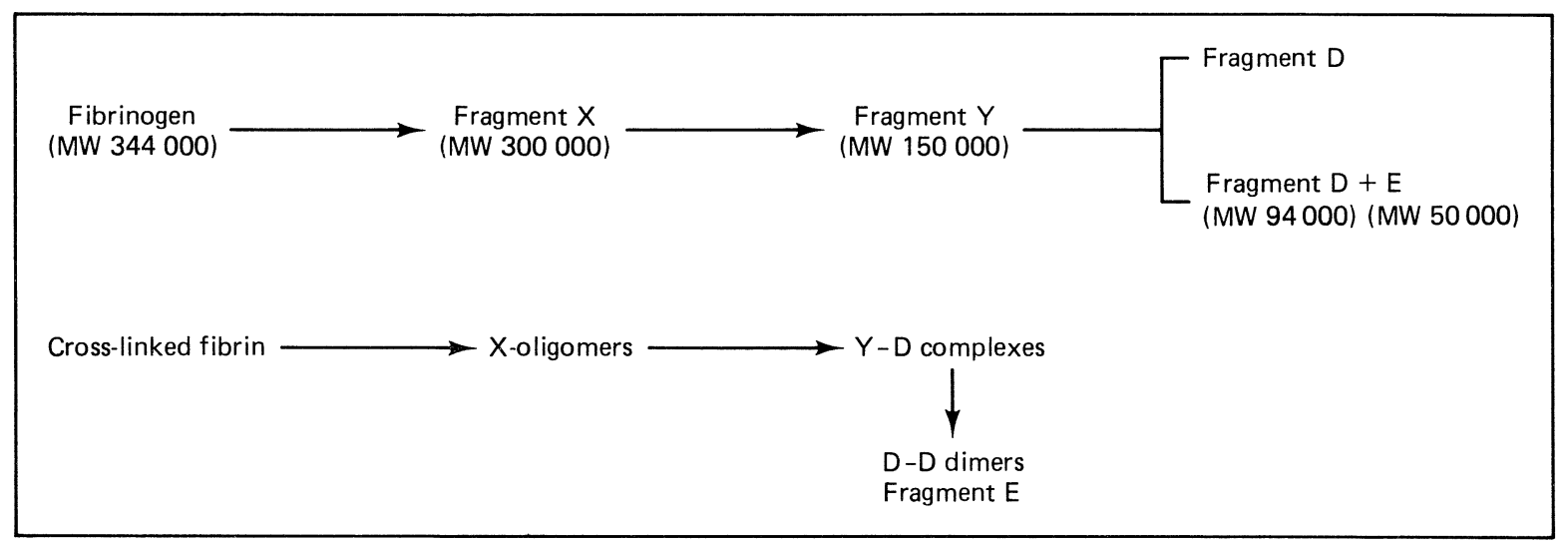

Fig. 42.1. Schematic representation of the progressive degradation products of fibrinogen and cross-linked fibrin.

occurs plasmin generation is initially rapidly neutralized by plasmin-alpha ${ }_{2}$-antiplasmin complexes. Following the decrease in noncomplexed alpha $_{2}$-antiplasmin, generalized free plasmin generation occurs, leading to lysis and breakdown of circulating fibrinogen as well as that of pre-formed cross-linked fibrin [27]. Progressive degradation of fibrinogen leads to the formation of the core fragments $D$ and $E$ via the intermediate fragments $X$ and $Y$. These reactions are simplistically shown in Fig. 42.1. The lysis of fibrin also leads to the formation of a variety of cross-linked complexes, including D-D dimers which differ antigenically from fibrinogen breakdown products. Raised levels of circulating fibrin complexes and fibrin/ fibrinogen degradation products (FDPs) inhibit the action of thrombin and inhibit platelet function by binding to the platelet membrane. Free plasmin also non-specifically cleaves a variety of peptides with arginyl-lysine bonds including factors $\mathrm{V}$ and VIII and the first component of complement.

Due to continual excessive fibrin formation liver cell synthesis of the coagulation factor proteins is unable to fully compensate for their consumption. This causes decreased circulating levels of all the coagulation factors but particularly of factors V, VIII, XIII and fibrinogen. Similarly bone marrow megakaryocyte production of platelets is unable to maintain a normal platelet count and thrombocytopenia results. A combination of coagulation factor deficiency, thrombocytopenia and the inhibitory actions of raised FDPs causes the generalized and continuing bleeding tendency. These pathogenic mechanisms are summarized in Fig. 42.2.

\section{Diseases Associated with DIC}

The main causes of DIC encountered in surgical practice in the UK are shown in Table 42.2. Approximately $60 \%$ of clinical cases of DIC are associated with a septicaemic infection, with Gram-negative bacteria being the most frequent organisms [65]. Severe haemorrhage and hypovolaemic shock may occur acutely in pregnancy,

Table 42.2. Main causes of DIC encountered in clinical practice

\begin{tabular}{ll}
\hline 1. Infections & $\begin{array}{l}\text { Septicaemia } \\
\text { Viraemia } \\
\text { Protozoal (malaria) }\end{array}$ \\
2. Malignancy & $\begin{array}{l}\text { Especially when } \\
\text { disseminated metastatic } \\
\text { carcinomas Leukaemia; } \\
\text { especially acute } \\
\text { promyelocytic } \\
\text { Septic abortion }\end{array}$ \\
& $\begin{array}{l}\text { Abruptio placentae } \\
\text { Eclampsia }\end{array}$ \\
3. Obstetric disorders & $\begin{array}{l}\text { Amniotic fluid embolism } \\
\text { Placenta praevia }\end{array}$ \\
& $\begin{array}{l}\text { Extensive surgical trauma } \\
\text { Burns }\end{array}$ \\
4. Shock & $\begin{array}{l}\text { Heatstroke } \\
\text { Cirrhosis } \\
\text { Acute hepatic necrosis }\end{array}$ \\
5. Liver disease & Tissue rejection \\
6. Transplantation & Cardiac bypass surgery \\
7. Extracorporeal & ABO incompatible \\
circulations & transfusion \\
8. Severe blood & \\
transfusion reaction & Certain snakebites
\end{tabular}




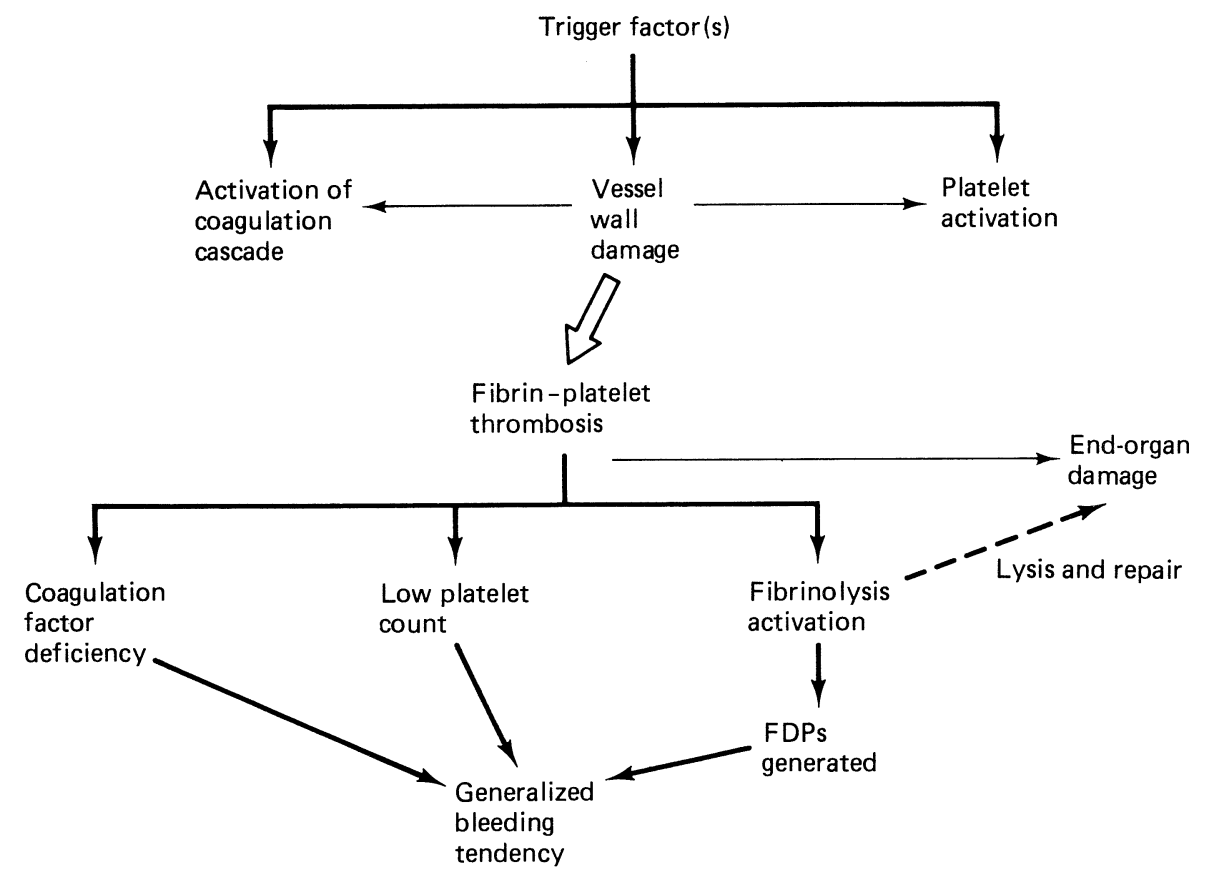

Fig. 42.2. Pathogenesis of acute DIC.

usually related to labour. During the later months of pregnancy there already exists a hypercoaguable state with increased activity of all the coagulation factors, particularly factor VII, a slight lowering of antithrombin III activity and decreased overall fibrinolytic activity. These changes are consistent with local placental activation of the coagulation system, with fibrin deposition occurring to some extent in normal pregnancy. Abruptio placentae, amniotic fluid embolism, intra-uterine fetal death and sepsis associated with abortion of premature rupture of the membranes are the most frequent obstetric emergencies to be complicated by acute life-threatening DIC [87]. In patients with abruptio placentae entrance of cellular elements of decidua and placenta with thromboplastinlike activity into the maternal circulation from the retroplacental area causes generalized coagulation and platelet activation. Similarly in amniotic fluid embolism the entrance of meconium and fetal squamous cells from the amniotic fluid into the maternal circulation activates the haemostatic system.

Malignancies of various types can lead to serious DIC. This is usually associated with metastatic disease, particularly with carcinoma of the pancreas, lungs and stomach [77]. DIC can also occur in all the various forms of leukaemia, but has a high incidence in associa- tion with the hypergranular form of acute promyelocytic leukaemia. It is triggered by the breakdown of the abnormal promyelocytic blast cells and the entrance of the thromboplastic granular material into the circulation following the initiation of cytotoxic chemotherapy during remission induction [37]. Following surgery for carcinoma of the prostate there is an increased risk of local urogenital and generalized bleeding. This is predominantly due to the release from prostatic tissue or metastatic lesions of material with plasminogen activator activity. Initially this causes local then systemic primary fibrinolysis, but secondary DIC may also be present.

\section{Laboratory Diagnosis of Acute DIC}

In the acute state of DIC it is essential to demonstrate the abnormal laboratory parameters accurately and quickly so that the appropriate therapy can be instigated [82]. Ideally the results of the screening tests should be available within $30 \mathrm{~min}$ after the laboratory receives the sample. To demonstrate depletion or consumption of clotting factors and platelets, the presence of intravascular haemolysis and the degree of the process the following simple tests should be performed: haemoglobin, platelet count, thrombin time, prothrombin time and an 
assessment of clottable fibrinogen. The main abnormalities are reflected by a prolonged thrombin time, hypofibrinogenaemia and thrombocytopenia. A fibrinogen level below 1.0 $\mathrm{g} /$ litre (normal range 1.5-4.0 g/litre) and a platelet count below $100 \times 10^{9} /$ litre are regarded as virtually diagnostic, especially if associated with a generalized bleeding state. Although patients will also have decreased levels of other coagulation factors, particularly factors II, V and VIII, specific factor assays, apart from that of fibrinogen, are of limited immediate value.

The thrombin time is prolonged by a deficiency of clottable fibrinogen, elevated FDP levels wich inhibit thrombin activity or the presence of circulating heparin. The thrombin time acts as the best practical guide to the clinical significance of raised fibrin degradation products (FDPs) and a low fibrinogen level. A prolonged thrombin time more than double the control time is usually indicative of impending overt clinical bleeding and support therapy should be instigated.

Secondary fibrinolytic activity causes raised levels of circulating fibrin complexes and FDPs. Plasma containing the earlier formed highmolecular-weight FDPs (fragments $X$ and $Y$ ) and fibrin complexes can be detected by the so-called paracoagulation tests which rely on gel formation after exposure to ethanol or protamine. These tests, although simple, are unreliable as non-specific positive reactions frequently occur. The provision of commercial kits to detect FDPs are now readily available and more reliable as rapid screening tests. Levels above $100 \mu \mathrm{g} / \mathrm{ml}$ are usually found in acute DIC (normal range less than $10 \mu \mathrm{g} / \mathrm{ml}$ ). However, some patients with severe DIC have no elevation of FDPs due to a general inhibition of their fibrinolytic response. These patients have a particularly poor prognosis due to irreversible organ failure. More sophisticated radioimmunoassays of specific FDP fragments (i.e. fragment $\mathrm{X}$ and $\mathrm{D}-\mathrm{D}$ dimers) have recently become available and may be useful in the diagnosis of the chronic compensated form of DIC without overt clinical symptoms [93].

The basic haemostatic abnormalities encountered in acute DIC have been outlined above. More sophisticated and time-consuming tests, often only available in the specialist haemostasis unit, may be useful in the diagnosis of the early stages of compensated subacute DIC before excessive haemorrhage occurs. Platelet activation can be detected by raised plasma levels of the platelet secretory products such as platelet factor $4, \beta$-thromboglobulin and thromboxane $\mathrm{A}_{2}$. Evidence of increased coagulation factor activation and thrombin generation is shown by raised levels of fibrinopeptide $A$ (the small peptide released from the N-terminal end of the A chains of fibrinogen initially by thrombin) and decreased levels of the two main inhibitory proteins antithrombin III and protein C.

Secondary activation of the fibrinolytic system is detected by depletion of plasminogen and alpha $_{2}$-antiplasmin levels and raised levels of the peptide $B \beta$ 15-42 (released by plasmininduced lysis of fibrin) and plasmin-antiplasmin complexes. Recently an acquired type II von Willebrand's disease defect has been described in patients with acute DIC with absence of the high-molecular-weight von Willebrand factor (vWf) multimers. This is probably caused by cleavage of the vWF multimers into smaller forms by excessive proteolytic enzyme activity with plasmin or calcium-activated proteases.

Reduced levels of plasma fibronectin also occur in acute DIC and fibronectin levels have been reported to correlate with elevated fibrin degradation products and reduced antithrombin III levels [64]. It is probable that fibronectin is deposited in the intravascular thrombi, through cross-linkage mediated by factor XIIIa with fibrin, and also has an increased consumption as a non-specific opsonin, expecially in septicaemic states. Decreased fibronectin levels further impair the clearance of infective organisms and other foreign material by the reticuloendothelial system and may impede correction of the pathogenic triggering factors.

\section{Management of Acute DIC}

Certain aspects of the management of acute DIC are extremely controversial and several widely different approaches are advocated. The first essential, if at all possible, is to eliminate the precipitating trigger factors. Wide-spectrum antibiotics (usually an aminoglycoside and a $\beta$ lactam penicillin) must be given intravenously and shock should be vigorously treated to maintain the blood volume and to avoid excess vascular stasis, hypoxia or acidosis. In obstetric situations rapid complete evacuation of the uterus may be life saving. However, in the presence of widespread uncontrollable bleeding specific replacement therapy should be immediately instigated.

Fresh frozen plasma (FFP) contains all the coagulation factors and the main inhibitors 
antithrombin III and protein C in near normal quantities, and four to five units (approximately $200 \mathrm{ml}$ per unit) should be rapidly infused [32]. FFP will also act as a valuable plasma expander. Cryoprecipitate which contains all components of the factor VIII complex as well as fibrinogen, factor XIII and fibronectin in concentrated form is a useful additional component and five to ten units should be infused initially along with the fresh frozen plasma. Platelet concentrates are the third essential blood component and five to ten units should also be infused as soon as they are available [70]. As soon as this initial replacement therapy with FFP, cryoprecipitate and platelet concentrates has been given a further blood sample should be taken to reassess their effect on the haemostatic process by repeating the simple screening tests. During this initial period packed red cells should be transfused to maintain the haematocrit above 0.30 and the blood volume maintained with human plasma protein fraction or crystalloid solution. These products do not contain any viable coagulation factors or platelets. The various factor IX (prothrombin complex) concentrates and specific fibrinogen preparations should not be used as they may potentiate the microvascular thrombotic tendency. In particular the various factor IX concentrates have been shown to contain variable amounts of activated coagulation factors including factors Xa and IXa which may enhance local fibrin deposition [33].

Following repetition of the haemostatic screening tests further replacement therapy should be judged by the response of the thrombin time, fibrinogen level, platelet count and degree of clinical bleeding. The use of heparin therapy in DIC remains controversial [21]. Reports of both benefit and marked deterioration are present in the literature. As a general guideline heparin should only be used after initial adequate replacement therapy has failed to control excessive bleeding. If used, a low-dose continuous intravenous regime using an automatic pump should be started at between 500 and 1000 units per hour with continuing replacement therapy. However, in certain specific indications clinical trials have shown early low-dose continuous heparin therapy to be clearly beneficial. Thess include before and during induction therapy for acute promyelocytic leukaemia, the early stages of amniotic fluid embolism and immediately a severe incompatible blood transfusion (i.e. an $\mathrm{ABO}$ mismatch) has been recognized to have occurred.
The use of fibrinolytic inhibitors, such as epsilon-aminocaproic acid (EACA) or tranexamic acid, should not be considered as this may result in the failure to remove fibrin thrombi from important organs such as the kidney.

During acute DIC there is also a rapid consumption of antithrombin III and protein C. These are the two main physiological inhibitors of inappropriate activation of the coagulation cascade. Antithrombin III inhibits mainly activated factor $X$ and thrombin, whilst heparin considerably augments this activity. Recent reports of specific replacement therapy with antithrombin III concentrates have been encouraging and should now be considered in severe cases if available [6]. In certain circumstances where platelet activation is the main pathogenic factor specific inhibition of platelet activation with an intravenous infusion of prostacyclin may be useful, particularly to maintain pulmonary and renal function.

\section{Liver Disease}

All the coagulation factors apart from factor VIII and von Willebrand protein are synthesized in the hepatocyte. The hepatocyte also synthesizes the main protease inhibitors antithrombin III, protein $C$, protein $S$, heparin cofactor II, and alpha $_{2}$-antiplasmin, and is responsible for the production of plasminogen, a pivotal protein in the fibrinolytic pathway. Furthermore, the liver is also involved in clearing the bloodstream of many activated intermediates of coagulation and fibrinolysis, a role largely undertaken by the reticuloendothelial cells lining the hepatic sinusoids. It is not surprising, therefore, that hepatocellular damage is frequently accompanied by complex disturbances of haemostasis and that the extent of these disturbances often reflects the severity of the underlying liver disease [15,74].

Bleeding is a common complication of both acute and chronic liver disease and frequently has a multifactorial aetiology. With the exception of factor VIII and von Willebrand protein, damage to the hepatocyte may result in reduced synthesis of all of the coagulation factors. In addition, concomitant cholestasis will result in malabsorption of fat-soluble vitamin $\mathrm{K}$. This vitamin is necessary for gamma-carboxylation of the glutamic acid residues of the precursors of factors II, VII, IX and X, without which they are 
unable to function effectively in the coagulation cascade. As factor $V$ is produced independently of the patient's vitamin $\mathrm{K}$ status, measurement of this coagulant protein may allow differentiation between the effects of pure vitamin $\mathrm{K}$ deficiency and the coagulopathy consequent on hepatocellular damage. Unlike many of the coagulant protein concentrations, that of fibrinogen is relatively well maintained in liver disease until the preterminal stage, when it may fall precipitously. However, at a much earlier stage, when liver function may be only mildly disturbed, the fibrinogen molecule produced by the damaged hepatocyte may have an abnormally high sialic acid content [23] and fails to function normally. This state of acquired dysfibrinogenaemia occurs frequently in chronic liver disease and primary hepatomas [24] and results in excessively slow fibrin polymerization following cleavage of fibrinopeptides $\mathrm{A}$ and $\mathrm{B}$ by thrombin and subsequently an unstable fibrin clot. By contrast with most other procoagulant proteins, factor VIII-von Willebrand complex is produced mainly outside the liver and their levels are characteristically raised in both acute and chronic hepatocellular failure. Factor VIII:C may be produced by the spleen and kidney as well as endothelial cells within the hepatic sinusoids and von Willebrand factor by the vascular endothelium and megakaryocytes. Indeed, the high plasma concentrations of factor VIII found in fulminant hepatitis contrast markedly with the low values characteristic of disseminated intravascular coagulation.

The essential role of the liver in synthesis of inhibitors of coagulation and clearance of activated products further complicates the coagulopathy of hepatic disease. Disseminated intravascular coagulation can be initiated or exacerbated by release of tissue thromboplastin from the damaged hepatocyte, reduced clearance of activated coagulation proteins, and reduced synthesis of the inhibitors heparin cofactor II, proteins $C$ and S, and antithrombin III. Although the plasma level of plasminogen falls in liver disease [84], reduced clearance of plasminogen activators has been demonstrated in cirrhotics [7] and this may result in an abnormal fibrinolytic response, which in turn would exacerbate a consumption coagulopathy. In spite of this, the DIC of liver disease alone is usually mild, although it may rapidly become life-threatening immediately after creation of a peritoneovenous shunt if large volumes of ascitic fluid are infused directly into the circulation [42]. This fluid has been shown to contain a potent thromboplastin-like procoagulant material [1] and for this reason ascitic fluid should be discarded at the time of the shunt insertion. Thrombocytopenia caused by hypersplenism or the presence of platelet-associated antibodies found in chronic active hepatitis and alcoholic cirrhosis $[4,41]$ may also occur.

The prothrombin time and activated partial thromboplastin time reflect the potential severity of the degree of liver cell failure. The thrombin time may be prolonged due to hypofibrinogenaemia, dysfibrinogenaemia, raised levels of fibrin degradation products, or the presence of heparin. The snake venom reptilase or defibrase (from Bothrops atrox) is a thrombin-like enzyme that only cleaves fibrinopeptide A (but not B) from fibrinogen and is unaffected by such antithrombins as heparin. The polymerization defect of fibrin that occurs when the fibrinogen molecule is abnormal is exaggerated by this enzyme and hence a prolonged reptilase clotting time in the face of a normal fibrinogen assay implies dysfibrinogenaemia, whilst correction of a long thrombin time is consistent with the presence of heparin. Fibrin degradation products may be elevated as a result of excessive fibrinolysis or because the liver fails to clear them from the bloodstream, whilst the factor VIII level may help to differentiate the effect of a consumptive coagulopathy from that of pure hepatocellular damage (see above).

Bleeding in liver disease is not necessarily related to the severity of the in vitro laboratory defects. For instance, a patient may have a major haemorrhage from oesophageal varices although the coagulation times may be near normal. The corollary is also true in that severe abnormalities of laboratory tests are not always associated with bleeding manifestations [74] and such patients who are not actively bleeding do not require replacement therapy. Nevertheless, percutaneous liver biopsy should not be routinely performed if either prothrombin or partial thromboplastin times are prolonged by more than $3 \mathrm{~s}$ over the control value.

Attempts to correct these abnormalities with $10 \mathrm{mg}$ of vitamin $\mathrm{K}_{1}$ given intravenously daily for three days should follow; if this proves unsuccessful then the biopsy should be performed under cover with fresh frozen plasma, preferably by the transvenous route [25]. Treatment of bleeding episodes associated with abnormal laboratory tests is largely based on replacement of clotting factors with fresh frozen plasma and platelet transfusions to maintain the platelet count above $50 \times 10^{9} /$ litre, although this 
will never completely correct the laboratory abnormalities and fluid overload must be avoided. Prothrombin complex (factors II, IX, and $\mathrm{X}$ ) concentrate may be used cautiously as an adjunct to FFP in severe cases, although care must be taken not to precipitate DIC as some concentrates contain appreciable amounts of activated factor $\mathrm{Xa}$ and thrombin [33]. The use of antifibrinolytic agents is not recommended as they may further promote intravascular coagulation whilst heparin has not been shown to be helpful in liver disease. In fulminant hepatitis antithrombin III concentrates have been shown to be beneficial [8], perhaps because they help protect against the inappropriate intravascular coagulation and further consumption of clotting factors that may occur in this condition. More recently the use of 1-deamino-8-d-arginine vasopressin (DDAVP) has been shown to reduce the bleeding and partial thromboplastin time in selected patients with cirrhosis [58].

\section{Liver Charcoal Perfusion}

Extracorporeal perfusion through activated charcoal columns coated with biocompatible polymers is commonly associated with the formation of platelet aggregates which lead to an increase of filtration pressure, thrombocytopenia, and the production of platelet function defects, which may compound the haemostatic disturbance. [92]. Prostacyclin administration may ameliorate some of the hemostatic alterations induced by charcoal haemoperfusion [14].

\section{Liver Transplantation}

In addition to the coagulopathy of any established hepatic disease, liver transplantation is associated with special haemostatic problems. The surgery is often technically difficult and may be complicated by bleeding from vascular anastomoses secondary to pre-existing portal hypertension and extensive fibrous adhesions from previous operations. The surgical procedure undoubtedly activates haemostatic mechanisms with resultant intravascular coagulation and this is complicated by markedly accelerated fibrinolysis, most notably due to impaired clearance of tissue-type plasminogen activator [18]. Heparinized preservative from the grafted liver may also exacerbate the coagulopathy. Management of these haemostatic defects involves the judicious use of fresh frozen plasma and platelet transfusions. Survival of the patient depends on the prompt synthesis of clotting factors and clearance of the activated components of coagulation and fibrinolysis by the newly grafted liver; if this does not occur the prognosis is dismal [9].

\section{Haemostatic Defects of Uraemia}

Bleeding is a serious complication of renal failure and may sometimes be fatal. Clinical manifestations include purpura, epistaxis, gastrointestinal bleeding, and occasionally haemorrhagic pericarditis and intracranial haemorrhage. The major haemostatic disturbance associated with uraemia is a defect in platelet function and the platelet/vessel wall interaction which is most readily assessed by the bleeding time [85]. Renal disease seldom results in deficiencies of coagulation factors unless complicated by uraemic enteritis (with concomitant malabsorption of vitamin K), liver disease or DIC.

Many qualitative platelet defects have been recorded in renal failure but perhaps the single most important determinant of uraemic bleeding is the low haematocrit that attends longstanding renal failure [75]. The prolonged bleeding time seen in these patients largely corrects following blood transfusion [46] and has also done so after administration of recombinant erythropoietin for the treatment of anaemia [63]. In addition, platelets have been shown to have impaired aggregation to adrenalin, collagen and ADP but these in vitro defects do not seem to correlate well with clinical bleeding in uraemic patients. Ristocetin-induced aggregation may also be impaired and this would suggest an abnormal interaction between platelets and von Willelrand factor (vWf). However, there is no convincing evidence in this setting of an abnormal vWf or, indeed, of the platelet membrane receptor for it, although some workers have been able to demonstrate proteolytic activity in uraemic blood which may enhance catabolism of vWf [49]. Furthermore, the ristocetin-induced aggregation response of normal platelets suspended in uraemic plasma is reduced [89] and this together with other data is compatible with the hypothesis that uraemic plasma may contain an inhibitor of the vWfplatelet interaction. DDAVP has been shown to reduce the bleeding time in uraemia [57] and 
is known to cause direct release of the factor VIII-vWf complex from endothelial cells; in this setting it may work by promoting a rise of plasma vWf (especially the larger multimers), thereby overcoming any inhibitor to the $\mathrm{vWf}-$ GP $1 b$ interaction [11].

Other qualitative abnormalities of platelets that have been documented include a storage pool defect with diminished serotonin and ADP content [19], reduced arachidonic acid metabolism associated with a functional cyclooxygenase defect [76], and increased platelet calcium content, correctable by vitamin $D$ analogues [29]. The precise uraemic retention products that are responsible for these platelet functional defects have not been identified, although so-called retained "middle molecules" of molecular weight between 500 and 3000 have been shown to inhibit platelet function in vitro as has parathormone, another possible uraemic toxin. It is interesting that whilst dialysis corrects the bleeding tendency of uraemic patients it does not always correct the platelet dysfunction.

Both peritoneal and haemodialysis will help reverse the haemostatic defect, although it may not entirely correct it. Indeed, haemodialysis may occasionally be associated with increased bleeding as platelets may be activated by the artificial membrane and anticoagulation is required to maintain patency of the extracorporeal circuit. For patients at high risk of bleeding heparin may be administered in low doses or may even be substituted by citrate or prostacyclin infusions [94]. Treatment of the anaemia to bring the haematocrit up to $30 \%$, either by blood transfusion or erythropoietin therapy, will improve platelet function and shorten the bleeding time [46]. Short-term correction of the haemostatic defect has been achieved with infusions of cryoprecipitate [34], but more recently DDAVP has been used successfully. Thus, the bleeding time has been reduced for up to $4 \mathrm{~h}$ following an intravenous infusion of $0.4 \mu \mathrm{g} / \mathrm{kg}$ of DDAVP [57] and this may allow minor surgery and renal biopsies to be performed safely. Finally, there is evidence that conjugated oestrogens may restore the bleeding time for some days in uraemic patients [47] although their mechanism of action is uncertain and it is not yet clear whether they reduce clinical bleeding in renal iailure.

\section{Renal Transplantation}

Some renal transplant patients with stable grafts have a prolonged bleeding time, even though in vitro platelet function tests are often unremarkable. Graft rejection may be accompanied by platelet consumption, and there is some evidence that the rejection process is mediated by microvascular thrombosis. Platelet activation may occur as a result of circulating immune complexes [10] and platelet membrane antigens have been localized to the glomeruli in renal biopsy specimens obtained from rejecting grafts. These findings emphasize the potential role of platelets in the rejection process and there is evidence that prostacyclin may be of benefit in this context [66].

\section{Coagulopathy Following Head Injury}

Disturbance of haemostasis following head injury may be due to stimulation of the extrinsic coagulation pathway by release of tissue thromboplastin, of which the brain is a particularly rich source, and to activation of the intrinsic pathway following damage to vascular endothelium. In addition, the effects of hypoxia, hypothermia, shock, and the release of catecholamines may all potentiate a consumption coagulopathy. Widespread microthrombi have been described in autopsy studies of patients dying following head injury, with the brain and lungs being frequent sites of involvement [40]. Experimental infusion of human FDPs into rabbits has produced a pathological state similar to the adult respiratory distress syndrome (ARDS) [51] and similar pulmonary dysfunction is common in head injury patients. It is probable that following severe head injury fibrin and fibrin degradation products may become trapped in the lung microvasculature and hence be a contributory factor in the complex pathophysiology of ARDS [79].

The reported incidence of disseminated intravascular coagulation following head injury varies from less than $2 \%$ [39] to greater than $70 \%$ [61] of cases. This wide range reflects not only the severity of head injury includeci in the series but, perhaps more importantly, the criteria used to define the presence of a consumptive coagulopathy. Thus, some studies rather loosely define DIC as being present if one 
or more coagulation tests are abnormal [61] whilst others use more specific criteria [88] and this makes direct comparison between different series difficult. Nevertheless, elevation of fibrin degradation products is the commonest single coagulation abnormality found in blunt head injury and in one series has been documented in 59 of $150(39 \%)$ such cases [90].

Not only has the degree of elevation of FDPs been shown to reflect the severity of the head injury [90] but also the haemostatic disturbance has been related to the incidence of delayed traumatic intracranial haematomas or haemorrhage, which may occur in up to $13 \%$ of cases $[39,88]$. More recently, Crone et al. [17] have presented data relating FDPs in patients with severe head injury to the development of earlyonset ARDS. Nine of 11 patients with FDP levels on admission greater than $64 \mu \mathrm{g} / \mathrm{ml}$ rapidly developed ARDS. By contrast, only 1 patient out of 22 with FDPs less than this level suffered this pulmonary complication. The authors suggest that a high level of FDPs may act as a sensitive indicator for the patient at risk of developing ARDS and should therefore prompt aggressive pulmonary therapy early on, with the use of positive end-expiratory pressure and haemodynamic monitoring as appropriate. The presence of a clinically significant consumption coagulopathy should be managed along conventional lines.

\section{Coagulopathy of Cardiopulmonary Bypass Surgery}

A wide variety of haemostatic defects have been described in association with cardiopulmonary bypass (CPB) surgery. These include thrombocytopenia, acquired platelet functional defects, coagulation factor deficiencies, increased fibrinolysis and fibrinogenolysis, inadequate heparin neutralization, and disseminated intravascular coagulation. In addition, haemodilution of coagulation factors by the fluid used to prime the extracorporeal circuit and the use of stored bank blood may further complicate the haemostatic picture. Despite all these in vitro disturbances, however, the coagulopathy of $\mathrm{CPB}$ is now seldom severe enough to result in excessive peri- or postoperative bleeding. In fact, most clinical bleeding problems arise in patients with added risk factors such as prolonged surgery (for multiple valve replacement or repeat coronary artery grafting), pre-existing liver dysfunction (e.g. secondary to tricuspid incompetence), or a raised haematocrit of over 0.55 (e.g. secondary to cyanotic heart disease).

Mild thrombocytopenia occurs in virtually all patients undergoing $\mathrm{CPB}$ surgery [5] but the platelet count seldom falls below $100 \times 10^{9}$ /litre unless the surgery is unduly prolonged. However, damage to the platelets as they pass through the pump and oxygenator system commonly causes a marked functional defect manifested by a prolonged bleeding time and associated with depletion of alpha granules and a reduced aggregation response to $\mathrm{ADP}[30,55]$. This defect usually corrects itself within $3 \mathrm{~h}$ following termination of bypass but should the defect persist and be associated with excessive haemorrhage then the patient should receive platelet concentrates even when the platelet count is greater than $100 \times 10^{9} /$ litre $[5,30]$.

The levels of coagulation factors following $\mathrm{CPB}$ surgery are frequently lower than preoperatively and may result in prolongation of the prothrombin and partial thromboplastin times [36]. This decrease in coagulation factors largely parallels the drop in haematocrit that occurs when extracorporeal circulation is instituted but in the absence of other haemostatic disturbances is seldom sufficiently severe to compromise haemostasis [55]. Of similar doubtful significance is the enhanced fibrinolytic activity that has been reported in many of these patients. This may result from activation of the plasminogen-plasmin system, perhaps by tissue-type plasminogen activator released from the vascular endothelium [86], or from reduction of the naturally occurring fibrinolytic inhibitors that occurs during bypass [71]. Whatever the reason, it seems unlikely that activation of fibrinolysis is sufficient to account for widespread bleeding [55]. The incidence of DIC, once commonly cited as a cause of bypassassociated bleeding, is probably very low $[3,55]$. Indeed, it is difficult to invoke a consumption coagulopathy as the cause of bleeding in the face of systemic heparinization during the bypass procedure.

Systemic heparinization is achieved with a loading dose of 200-300 units of heparin per kilogram followed by additional doses as required. Control of the dose is usually performed in theatre by means of the activated clotting time (ACT) using the Hemocron, the aim being to keep the ACT between 400 and $600 \mathrm{~s}$. The 
patient's sensitivity to heparin may be assessed by constructing a dose-response curve (i.e. heparin dose versus ACT) and this may subsequently be used to calculate the amount of protamine required to reverse anticoagulation at the end of bypass. The protamine is given as a slow infusion and, as a guide, $1 \mathrm{mg}$ of protamine neutralizes 100 units of heparin. Alternatively, a dose of $2-3 \mathrm{mg} / \mathrm{kg}$ may be administered regardless of total amount of heparin received during the procedure. This appears to be adequate in virtually all cases [20] without inducing an anticoagulant effect of itself. In theory, heparin rebound may occur some hours after the end of bypass when the anticoagulant, previously sequestered in the tissues, re-enters the circulation and also possibly from the release of heparin from bound protamine-heparin complexes. In practice, however, this seldom occurs with today's doses of heparin and protamine and therefore is very rarely responsible for postoperative bleeding $[5,13]$.

Identification of high-risk patients prior to surgery allows appropriate measures to be taken in advance. Thus, platelet concentrates may be made available for patients who are likely to have prolonged surgery or who have already developed a platelet functional defect secondary, perhaps, to recent ingestion of a non-steroidal anti-inflammatory drug. If a preexisting coagulopathy is found then this should be investigated and treated if possible (e.g. with vitamin K). Excessive bleeding post-bypass should be managed in the light of simple laboratory test. In view of the functional defect sustained by the platelets, platelet concentrates should be given even in the face of a relatively normal count, and certainly if they are reduced to below $100 \times 10^{9} /$ litre. Fresh frozen plasma and/or cryoprecipitate is appropriate if haemodilution has been sufficient to result in convincing prolongation of laboratory coagulation times.

There have been several recent attempts to reduce blood loss from cardiac surgery using pharmacological agents. Prostacyclin has been shown to increase platelet numbers at the end of bypass but does not seem to reduce transfusion requirements [22]. More encouraging, however, has been the study by Salzman et al. [80] in which desmopressin (1-deamino-8-darginine vasopressin, DDAVP) was given as a short infusion at the end of bypass to 35 patients and found to reduce mean blood loss from 2210 $\mathrm{ml}$ in control patients to $1317 \mathrm{ml}$ in the study patients. The mechanism of action of DDAVP in this context is unclear, but there is recent evidence that it may cause the vWf to exceed a threshold concentration necessary for effective platelet-vessel wall interaction [91]. Even more impressive are the data reported by Royston and colleagues [78], who studied 11 patients undergoing repeat open heart surgery with the serine proteinase aprotinin. This was given as a continuous infusion throughout the procedure and reduced the mean blood loss from $1509 \mathrm{ml}$ in the control group to only $286 \mathrm{ml}$ in the study group. In neither the DDAVP nor the aprotinin study were patients undergoing uncomplicated first-time coronary artery bypass surgery included. It remains to be seen whether these agents may safely be used in such patients in whom postoperative graft thrombosis must at all costs be avoided.

\section{Vitamin K Deficiency in Gastrointestinal Disorders}

Development of vitamin $\mathrm{K}$ deficiency in otherwise healthy individuals is rare. Not only is the daily requirement low (about $0.1-0.5 \mu \mathrm{g} / \mathrm{kg}$ per day) but the vitamin is to be found in most foods and is also synthesized by gut bacteria, some of which is absorbed through the terminal ileum. Deficiency of this vitamin results most commonly from drug therapy or disease states, some of which are listed in Table 42.3.

It normally takes several weeks for a coagulopathy to develop in healthy subjects fed a diet low in vitamin K. However, this occurs much

Table 42.3. Causes of vitamin $\mathrm{K}$ deficiency

1. Haemorrhagic disease of the newborn

2. Dietary inadequacy (low-fat diets, protein-calorie malnutrition)

3. Total parenteral nutrition

4. Biliary obstruction (strictures, stones, fistulas)

5. Malabsorption states (coeliac disease, sprue, ulcerative colitis, regional ileitis, etc.)

6. Liver disease

7. Drugs

Coumarins (warfarin, phenindione)

Hydantoins (phenytoin)

Salicylates

Broad-spectrum antibiotics (including cephalosporins) Large doses of vitamins $\mathrm{A}$ and $\mathrm{E}$ 
more rapidly if the subject is debilitated or receiving antibiotics which alter the bowel microflora. Patients who have a poor food intake after surgery, or who are receiving antibiotics, may develop haemorrhagic manifestations after only two weeks [2,72]. Similarly, patients on parenteral nutrition may become vitamin $\mathrm{K}$ deficient as physiological doses of the fat-soluble vitamins are not metabolized normally when they are introduced directly into the bloodstream. In these circumstances at least $1 \mathrm{mg}$ of additional vitamin $\mathrm{K}$ should be supplied each week [69]; in the face of increased requirements this may need to be increased to $10 \mathrm{mg}$ weekly to maintain a normal prothrombin time. Commercial preparations of fat-soluble vitamins may not be sufficient to meet this demand (e.g. Vitlipid supplies $0.15 \mathrm{mg}$ of vitamin K) and consideration should also be given to providing extra folic acid and vitamin $\mathrm{C}$, deficiencies of which may exacerbate any pre-existing coagulopathy.

Other conditions that predispose to vitamin $\mathrm{K}$ deficiency include any cause of biliary obstruction, malabsorption syndromes consequent of small bowel pathology, liver disease and drug therapy. Certain parenterally administered broad-spectrum cephalosporins (e.g. moxalactam) may interfere with vitamin K-dependent gamma-carboxylation of the clotting factors within the liver [45]. The anticoagulants have a predictable effect, but orally administered antibiotics may alter the ileal microflora with consequent reduction in the endogenously synthesized vitamin $K$. Thus, patients who receive bowel-sterilizing antibiotics prior to gut surgery may develop bleeding manifestations postoperatively, especially if they are on a restricted diet as well, as may patients with biliary obstruction. Vitamin $\mathrm{K}$ should be given prophylactically to these patients. The recommended dose is $10 \mathrm{mg}$ daily for three days before surgery (including liver biopsy). A similar trial of vitamin $\mathrm{K}$ therapy may be given to patients with hepatocellular disease, although the coagulopathy is unlikely to respond unless there is an element of malabsorption.

\section{Haemostatic Defects Associated with Massive Transfusions}

When the patient's blood volume is replaced by the administration of large quantities of stored banked blood in a short period of time, haemorrhagic manifestations are liable to follow [13]. Stored whole blood contains a progressive loss of mainly factors V and VIII with a decrease in factor VIII activity to approximately $50 \%$ after one day, 30\% after five days, $6 \%$ after 21 days and factor $\mathrm{V}$ factor falling to $50 \%$ after 14 days [16]. There is no appreciable loss in the other coagulation factors on storage for 21 days. Platelet function, however, is quickly lost on storage and after $48 \mathrm{~h}$ storage there are practically no viable functioning platelets. If plasmareduced red cells or red cells collected into an optimal additive solution are transfused, further dilution of coagulation factors will occur. Obviously additional infusion of crystalloids, human albumin preparations or one of the artificial colloid substitutes will also cause further dilution of coagulation factors and platelets. The various red cell preparations may also precipitate disseminated intravascular coagulation owing to a combination of partial activation of clotting factors and breakdown of platelets, leucocytes and red cells releasing thromboplastin-like material during storage. There is also evidence that platelet function may be impaired in the massively transfused patient.

Previously standardized schemes of administering platelet concentrates and fresh frozen plasma have been followed after the equivalent replacement of one blood volume (approximately 10 units in adults) [26]. However, to prevent indiscriminate use of components, massive transfused patients should have routine haemostasis tests monitored early to define the precise abnormality [56]. Such patients primarily develop microvascular bleeding with oozing from mucosa, raw wounds and puncture sites due to thrombocytopenia with platelet counts $<50 \times 10^{9} /$ litre when approximately 1.5-2 times their blood volume has been transfused. Thrombocytopenia can also be accentuated in these circumstances by accelerated platelet utilization. A standard dose of 6 units of platelet concentrates should be infused to control microvascular bleeding when this occurs. Generally the haemostatic levels of the coagulation factors are well maintained and fresh frozen plasma is not required prophylactically [35]. The use of fresh frozen plasma should be restricted to defined defects in the coagulation cascade with prolongation of the prothrombin time by $5 \mathrm{~s}$ or more.

To maintain blood volume during the acute stages of hypovolaemic shock large volumes of synthetic colloids such as dextrans, gelatin 
solutions or hydroxyethyl starch or human albumin solutions are frequently infused [53]. In addition to their simple dilutional effect on all the coagulation factors and platelet count they may further interfere and inhibit haemostasis to variable degrees. Dextran has been shown to accelerate the action of thrombin on fibrinogen (thus shortening the thrombin time) to reduce the plasma levels of factor VIII and vWf and induce an acquired von Willebrand's disease, and due to adsorption onto the platelet surface cause decreased platelet adhesiveness and aggregation by steric blockage of the surface membrane receptors [62]. These defects are additive and dose dependent and may significantly prolong the bleeding time and activated partial thromboplastin time, and predispose to clinical bleeding episodes. Similarly hydroxyethyl starch due to its macromolecular properties will induce a slight shortening of the thrombin time and similar factor VIII defects in vitro [54], but these effects are of negligible clinical significance with infused volumes of up to $1500 \mathrm{ml}$ [48]. Human albumin solutions infused in large volumes have also been shown to further inhibit haemostasis, possibly by decreasing hepatic protein synthesis or by the presence of prekallikrein activator in the infused solution stimulating fibrinolysis [50]. The modified gelatins, although adsorbed onto the platelet surface, do not induce any inhibitory haemostatic defect. However, gelatin solutions have been shown to significantly decrease plasma fibronectin levels and inhibit fibronectin function by gelatin-fibronectin interactions. This may potentially decrease the opsonization of noxious material and subsequent phagocytosis by the reticuloendothelial system and delay early wound healing and cross-linkage of fibrin by factor XIIIa.

\section{Complications of Anticoagulant and Thrombolytic Drugs}

\section{Warfarin}

By far the most important complication of warfarin therapy is haemorrhage. However, this rarely occurs in the absence of underlying pathology if the dose of warfarin is controlled by regular international normalized ratio (INR) estimations derived from the prothrombin time (PT). The INR is a ratio that relates the sensitiv- ity of the thromboplastin reagent used in the PT test to that of a World Health Organization standard, and this enables the results of PTs derived from different thromboplastin reagents to be directly compared. Depending on the indications for anticoagulation, the INR should be kept between 2.0 and 4.5 .

The management of a patient who is found to be over-anticoagulated largely depends on whether or not that patient is bleeding. In the absence of haemorrhage it is usually sufficient to stop warfarin therapy for a few days if the INR is significantly prolonged above the therapeutic range. A small dose of vitamin K (1-2.5 $\mathrm{mg}$ ) may be given orally or intravenously if the INR is greater than 7.0, as the risk of dangerous haemorrhage is proportionately greater at this level. Reversal of anticoagulation should certainly be instigated if the patient is bleeding: fresh frozen plasma $(10-15 \mathrm{ml} / \mathrm{kg})$ will immediately supply the missing coagulation factors, whilst vitamin $\mathrm{K}$ therapy will not be fully effective for at least $24 \mathrm{~h}$ and perhaps longer. Furthermore, doses of greater than $10 \mathrm{mg}$ of vitamin $\mathrm{K}$ may render the patient insensitive to further warfarin for some days or weeks and therefore should not be used if continued warfarin therapy is anticipated. Nevertheless, life-threatening bleeding should be treated with at least $50 \mathrm{mg}$ of vitamin $\mathrm{K}$ given intravenously and supplemented with FFP as a source of immediate clotting factors. Prothrombin complex concentrates are seldom used to reverse coumarin-induced anticoagulation as there is a risk of transmission of the AIDS and hepatitis viruses, and of thromboembolism from activated components found in the concentrate. Haemorrhage occurring in a patient whose warfarin dose is well controlled (INR $<4.5$ ) should be managed along the above lines but, in addition, the patient should be investigated for a possible underlying lesion to account for the bleed. The discovery of an occult large bowel neoplasm may often occur after beginning anticoagulant therapy.

Surgery may usually be performed on patients receiving warfarin provided that the prothrombin time is not greatly prolonged. It is recommended by the British Society of Haematology that the INR be between 2.0 and 2.5 for effective prophylaxis against venous thrombosis during high-risk surgery (e.g. hip and pelvic operations) although many surgeons are reluctant to operate if the INR is greater than 2.0. However, full reversal of warfarin effect is mandatory in patients undergoing eye or brain/ 
spinal cord surgery. Patients receiving longterm anticoagulation for cardiac and other prostheses should stop warfarin therapy three days prior to elective surgery. Specific reversal with vitamin $\mathrm{K}$ is usually not indicated because of the subsequent refractory state to further postoperative warfarin that this induces. However, if the prothrombin time performed $48 \mathrm{~h}$ before surgery is still greatly prolonged then a small dose (e.g. $1 \mathrm{mg}$ ) of vitamin $\mathrm{K}$ may be indicated. The PT should be repeated on the day of surgery and if still unacceptably prolonged then FFP administered. Although non-cardiac operations have been successfully performed on such patients in whom anticoagulation has been completely reversed [38], it is probably advisable to administer low-dose subcutaneous heparin (5000 u b.d.) preoperatively whilst the patient is off warfarin. This dose will not affect the prothrombin time estimation. Postoperatively, full-dose heparin should be given intravenously as soon as the immediate risk of perioperative bleeding is past (e.g. $12 \mathrm{~h}$ following surgery) and continued until the patient is once again anticoagulated with warfarin.

\section{Heparin}

Intravenous heparin has a short half-life of about $90 \mathrm{~min}$ and the anticoagulant effect may be monitored by the partial thromboplastin time or thrombin time. Minor haemorrhage associated with an infusion of heparin may usually be controlled by reduction of the administered dose or, if necessary, complete cessation of heparin therapy for a few hours. If bleeding is severe intravenous protamine may be used to immediately neutralize circulating heparin. One milligram of protamine neutralizes $100 \mathrm{u}$ of heparin. In practice it is often difficult to accurately assess the protamine dose in advance. It is usual, therefore, to give a small dose of protamine (e.g. $10 \mathrm{mg}$ ) and to repeat the coagulation tests afterwards. Further doses may be titrated against the results of these tests. If given in excess, protamine can exert an anticoagulant effect of its own and hence it is important that careful laboratory monitoring be performed in this setting.

Heparin-induced thrombocytopenia is now a well recognized complication of both short- and long-term therapy [12]. There seem to be two mechanisms by which this occurs. A nonspecific platelet-aggregating effect, possibly mediated through fibrinogen, commonly results in thrombocytopenia within a few days of starting heparin therapy. The platelet count seldom falls below $80 \times 10^{9}$ /litre and rapidly returns to normal after heparin has been stopped. Haemorrhage is unusual and clinical problems seldom arise. More seriously, but less commonly, heparin may induce an immunologically mediated thrombocytopenia that occurs at least one week after starting treatment. Thrombocytopenia may be severe, with platelet counts of $30 \times 10^{9} /$ litre or less and associated with arterial thrombosis. In these circumstances heparin should be stopped immediately and an alternative form of anticoagulation commenced. If severe, thrombolytic therapy may be required.

\section{Thrombolytic Agents}

As with the anticoagulants, the major complication of fibrinolytic therapy is haemorrhage. All the thrombolytic agents induce a state of systemic lysis, although the degree to which this is effected varies with the particular agent involved. Streptokinase (SK) has a greater effect on the laboratory indicators of systemic lysis than tPA and might, therefore, by expected to be associated with untoward haemorrhage more frequently than tPA. However, there is very little correlation between the incidence of bleeding and the degree of disturbance of laboratory tests and in practice tPA has not always been found to be significantly safer in this respect than SK [73]. It would appear that bleeding following fibrinolytic therapy results from the dissolution of haemostatic plugs with consequent exposure of the underlying damaged vessel wall before healing has occurred; a systemic lytic state exacerbates rather than causes haemorrhage from these sites.

Untoward haemorrhage complicating fibrinolytic therapy is most commonly local; it often occurs at the catheterization site in the groin or following venepuncture for right heart haemodynamic studies, although it may sometimes present from a site distant to that of trauma (e.g. gastrointestinal haemorrhage). Attention to careful patient selection, avoidance of invasive procedures, and limitation of the duration of therapy reduces the overall incidence of haemorrhagic complications to around 5\% [67] and it is clear that the more prolonged the thrombolytic therapy the greater the risk of excessive bleeding. Thus, whilst $1 \%$ of patients treated for $12 \mathrm{~h}$ or longer with a thrombolytic agent may 
suffer an intracranial haemorrhage [67] only $0.2 \%$ were found to do so when given a $1 \mathrm{~h}$ infusion of SK in the GISSI study [28].

Laboratory monitoring of thrombolytic therapy is of limited use as it seldom predicts the likelihood of haemorrhage and many thrombolytic regimens now employ fixed dosage schedules. Nevertheless, the thrombin time is sensitive to low fibrinogen levels and the inhibitory effects of fibrin(ogen) degradation products, and may be used to document the presence of a systemic lytic state following initiation of therapy [59]. Laboratory tests may demonstrate no change in $5 \%-10 \%$ of cases receiving fixed-dose SK therapy and these patients may be resistant to this thrombolytic agent.

Excessive haemorrhage from a local site may be controlled by local measures (e.g. pressure packing) with transfusion of blood if required. Serious bleeding may necessitate cessation of thrombolytic therapy; the fibrinolytic state reverses within a few hours as the plasma halflife of all agents used is short (i.e. minutes). The only exception to this is the recently employed agent APSAC (acylated plasminogenstreptokinase activator complex), which has a half-life of $90 \mathrm{~min}$. If haemorrhage is lifethreatening then the hypocoagulable state may be reversed with an infusion of fresh frozen plasma or cryoprecipitate. The efficacy of antifibrinolytic drugs in this context has not been proven although they would be a logical addition in the management of intracranial haemorrhage [60].

Other complications of thrombolytic therapy include partial lysis of clots with subsequent embolization downstream of the thrombus. This may, for example, result in stroke if the source of the embolus is the left ventricle or an ischaemic limb if the embolus originates from a peripheral artery [83]. Allergic reactions to SK may occur (due to antistreptococcal antibodies already present) but is seldom severe and may be controlled by corticosteroids and antihistamines; such reactions to other thrombolytic agents are rare.

\section{References}

1. Addonizio VP, Fisher CA, Strauss JF et al. (1987) Preliminary characterization of the procoagulant material in human ascites. Surgery 101:753-762
2. Ansell JE, Kumar R, Deykin D (1977) The spectrum of vitamin K deficiency. JAMA 238:40-42

3. Bachmann F, McKenna R, Cole ER, Najafi H (1975) The hemostatic mechanism after open-heart surgery. I. Studies on plasma coagulation factors and fibrinolysis in 512 patients after extracorporeal circulation. J Thorac Cardiovasc Surg 70:76-85

4. Barrison IG, Knight ID, Viola L et al. (1981) Platelet associated immunoglobulins in chronic liver disease. $\mathrm{Br}$ J Haematol 48:347-350

5. Bick RL (1985) Hemostasis defects associated with cardiac surgery, prosthetic devices, and other extracorporeal circuits. Semin Thromb Hemostasis 11:249-280

6. Blauhut B, Kramar H, Vinazzer H, Bergmann H (1985) Substitution of antithrombin III in shock and DIC: a randomized study. Thromb Res 39:81-85

7. Booth NA, Anderson JA, Bennett B (1984) Plasminogen activators in alcoholic cirrhosis: demonstration of increased tissue type and urokinase type activator. J Clin Pathol 37:772-777

8. Braude S, Arias J, Hughes RD et al. (1981) Antithrombin III infusion during fulminant hepatic failure. Thromb Haemostasis 46:369

9. Calne RY (1987) Management of bleeding. In: Calne RY (ed) Liver transplantation: The Cambridge/King's College Hospital experience, 2nd ed. Harcourt Brace Jovanovich, London, pp 247-250

10. Capitano A, Mannucci PM, Ponticelli C, Pareti F (1982) Detection of circulating released platelets after renal transplantation. Transplantation 33:298-301

11. Castaldi PA, Gorman DJ (1987) Disordered platelet function in renal disease. In: Colman RW, Hirsh J, Marder VJ, Salzman EW (eds) Hemostasis and thrombosis: basic principles and clinical practice. Lippincott, Philadelphia, pp 960-964

12. Chong BH (1988) Heparin-induced thrombocytopenia. Blood Rev 2:108-114

13. Collins JA (1976) Massive transfusion. Clin Haematol 5:201-227

14. Cordopatri F, Boncinelli S, Marsili M et al. (1982) Effects of charcoal hemoperfusion with prostacyclin on the coagulation-fibrinolysis system and platelets of patients with fulminant hepatic failure: preliminary observations. Int J Artif Organs 5:243-247

15. Cordova C, Violi F, Alessandri C et al. (1986) Prekallikrein factor VII as prognostic index of liver failure. Am J Clin Pathol 85:579-582

16. Counts RB, Haisch C, Simon TL et al. (1979) Haemostasis in massively transfused trauma patients. Ann Surg 190:91-99

17. Crone KR, Lee KS, Kelly DI (1987) Correlation of admission fibrin degradation products with outcome and respiratory failure in patients with severe head injury. Neurosurgery 21:532-536

18. Dzik WH, Arkin CF, Jenkins RL, Stump DC (1988) Fibrinolysis during liver transplantation in humans: role of tissue-type plasminogen activator. Blood 71 : 1090-1095

19. Eknoyan G, Brown CH (1981) Biochemical abnormalities of platelets in renal failure: evidence for decreased platelet serotonin, adenosine diphosphate, and $\mathrm{Mg}$ dependent adenosine triphosphatase. Am J Nephrol 1:17-23

20. Esposito RA, Culliford AT, Colvin SB, Thomas SJ, Lackner H, Spencer FC (1983) The role of the activated clotting time in heparin administration and neutralization for cardiopulmonary bypass. J Thorasc Cardiovasc Surg 85:174-185 
21. Feinstein DI (1982) Diagnosis and management of disseminated intravascular coagulation: the role of heparin therapy. Blood 60:284-288

22. Fish KJ, Sarnquist FH, van Steenis C et al. (1986) A prospective, randomized study of the effects of prostacyclin on platelets and blood loss during coronary bypass operations. J Thorac Cardiovasc Surg 91:436-442

23. Flute PT (1977) Disorders of plasma fibrinogen synthesis. Br Med Bull 33:253-259

24. Francis JL, Armstrong DJ (1982) Acquired dysfibrinogenaemia in liver disease. J Clin Pathol 35:667-672

25. Gazzard BG, Henderson JM, Williams R (1975) The use of fresh frozen plasma or a concentrate of factor IX as replacement therapy before liver biopsy. Gut 16: $621-625$

26. Gill W, Champion HR, Lang WB, Austin EA, Cowley RA (1975) Volume resuscitation in critical major trauma. J R Coll Surg Edinburgh 20:166-173

27. Graeff H, Hafter R (1982) Detection and relevance of crosslinked fibrin derivatives in blood. Semin Thromb Haemostasis 8:57-71

28. Gruppo Italiano per lo Studio Della Streptochinasi Nell'infarto Miocardico (1986) Effectiveness of intravenous thrombolytic treatment in acute myocardial infarction. Lancet $\mathrm{i}: 397-402$

29. Gura V, Creter D, Levi J (1982) Elevated thrombocyte calcium content in uremia and its correction by 1 alpha $(\mathrm{OH})$ vitamin D treatment. Nephron 30:237-239

30. Harker LA, Malpass TW, Branson HE, Hessel EA, Slichter SJ (1980) Mechanism of abnormal bleeding in patients undergoing cardiopulmonary bypass: acquired transient platelet dysfunction associated with selective alpha-granule release. Blood 56:824-834

31. Harker LA (1986) Bleeding after cardiopulmonary bypass. N Engl J Med 314:1446-1448

32. Hehne HJ, Nyman HB, Bussi H, Wolff G (1979) Management of bleeding disorders in traumatic haemorrhagic shock states with fresh frozen plasma. Eur J Intensive Care Med 2:157-161

33. Hultin MB (1979) Activated clotting factors in factor IX concentrates. Blood 54:1028-1033

34. Janson PA, Jubelirer SJ, Weinstein MS, Beykin D (1980) Treatment of bleeding tendency in uremia with cryoprecipitate. N Engl J Med 303:1318-1322

35. Jones J (1987) Abuse of fresh frozen plasma. Br Med J 295:287

36. Kalter RD, Saul CM, Wetstein L, Soriano C, Reiss RF (1979) Cardiopulmonary bypass: associated hemostatic abnormalities. J Thorac Cardiovasc Surg 77:427-435

37. Kantarjian HM, Keating MJ, Walters RS (1986) Acute promyelocytic leukaemia. Am J Med 80:789-799

38. Katholi RE, Nolan SP, McGuire LB (1978) The management of anticoagulation during noncardiac operations in patients with prosthetic heart valves: a prospective study. Am Heart J 96:163-165

39. Kaufmann HH, Moake JL, Olson JD et al. (1980) Delayed and recurrent intracranial hematomas related to disseminated intravascular clotting and fibrinolysis in head injury. Neurosurgery 7:445-449

40. Kaufmann HH, Hui K-S, Mattson JC et al. (1984) Clinicopathological correlations of disseminated intravascular coagulation in patients with head injury. Neurosurgery 15:34-42

41. Landolfi R, Leone G, Fedeli G et al. (1980) Plateletassociated $\operatorname{IgG}$ in acute and chronic hepatic diseases. Scand J Haematol 25:417-422

42. LeVeen HH, Ahmed N, Hutto RB, Moon I, LeVeen EG
(1987) Coagulopathy post peritoneovenous shunt. Ann Surg 205:305-311

43. Lindsay RM, Friesen $M$, Koens $F$, Linton $A L$, Oreopoulos D, de Veber G (1976) Platelet function in patients on long-term peritoneal dialysis. Clin Nephrol 6:335-339

44. Lindsay RM, Dennis BN, Bergstrom JC, Jonsson C, Furst P (1981) Platelet function as an assay for uremic toxins. Artif Organs 4(suppl):82-89

45. Lipsky JJ (1983) N-Methyl-thio-tetrazole inhibition of the gamma carboxylation of glutamic acid: possible mechanisms for antibotic-associated hypoprothrombinaemia. Lancet ii:192-193

46. Livio M, Gotti E, Marchesi D, Remuzzi G, Mecca G, de Gaetano G (1982) Uraemic bleeding: role of anaemia and beneficial effect of red cell transfusions. Lancet ii: 1013-1015

47. Livio M, Mannucci PM, Vigano G et al. (1986) Conjugated oestrogens for the management of bleeding associated with renal failure. N Engl J Med 315:731-735

48. Lockwood DNJ, Bullen C, Machin SJ (1988) A severe coagulopathy following volume replacement with hydroxyethyl starch in a Jehovah's Witness. Anaesthesia 43:391-393

49. Lombardi R, Mannucci PM, Seghatchian MJ, Garcia VV, Coppola R (1981) Alterations of factor VIII von Willebrand factor in clinical conditions associated with an increase in its plasma concentration. $\mathrm{Br} \mathrm{J}$ Haematol 49:61-68

50. Lucas CE, Ledgerwood AM, Mammen EP (1982) Altered coagulation protein content after albumen resuscitation. Ann Surg 197:198-202

51. Luterman A, Manwaring D, Curreri PW (1977) The role of fibrinogen degradation products in the pathogenesis of the respiratory distress syndrome. Surgery 82:703709

52. Machin SJ, Mackie IJ (1989) In: Laboratory haematology. Churchill Livingstone, Edinburgh

53. Macintyre E, Bullen C, Machin SJ (1985a) Fluid replacement in hypovolaemia. Intensive Care Med 11:231233

54. Macintyre E, Mackie IJ, Ho D, Tinker I, Bullen C, Machin SJ (1985b) The haemostatic effects of hydroxyethyl starch (HES) used as a volume expander. Intensive Care Med 11:301-304

55. Mammen EF, Koets MH, Washington BC et al. (1985) Hemostasis changes during cardiopulmonary bypass surgery. Semin Thromb Hemostasis 11:281-292

56. Mannucci PM, Federici AB, Sirchia G (1982) Hemostasis testing during massive blood replacement. Vox Sang 42:113-123

57. Mannucci PM, Remuzzi G, Pusineri F et al. (1983) Deamino-8-D-arginine vasopressin shortens the bleeding time in uremia. N Engl J Med 308:8-12

58. Mannucci PM, Vicente V, Vianello L et al. (1985) Controlled trial of desmopressin in liver cirrhosis and other conditions associated with a prolonged bleeding time. Blood 67:1148-1153

59. Marder VJ, Bell WR (1987) Fibrinolytic therapy. In: Colman RW, Hirsh J, Marder VJ, Salzman EW (eds) Hemostasis and thrombosis: basic principles and clinical practice. Lippincott, Philadelphia, pp 1393-1437

60. Marder VJ, Sherry S (1988) Thrombolytic therapy: current status (Part I). N Engl J Med 318:1512-1520

61. Miner ME, Kaufmann HH, Graham SH, Haar FH, Gildenberg PI (1982) Disseminated intravascular coagulation fibrinolytic syndrome following head injur $y$ 
in children: frequency and prognostic implications. J Paediat 100:687-691

62. Mischler JM (1984) Synthetic plasma volume expanders: their pharmacology, safety and clinical efficacy. Clin Haematol 13:75-92

63. Moia M, Mannucci PM, Vizzotto L, Casati S, Cattaneo M, Ponticelli C (1987) Improvement in the haemostatic defect of uraemia after treatment with recombinant human erythropoietin. Lancet ii:1227-1229

64. Mosher DF, Williams EM (1978) Fibronectin concentration is decreased in plasma of severely ill patients with disseminated intravascular coagulation. J Lab Med 91:729-737

65. Muller-Berghaus M (1987) Septicaemia and vessel wall In: Verstraete M, Vermylen J, Lijnen R, Arnout J (eds) Thrombosis and haemostasis 1987. University Press, Leuven, pp 619-671

66. Mundy AR, Bewick M, Moncada S, Vane JR (1980) Short term suppression of hyperacute renal allograft rejection in presensitized dogs with prostacyclin. Prostaglandins 19:595-603

67. National Institutes of Health Consensus Panel (1980) Thrombolytic therapy in thrombosis: a National Institutes of Health consensus development conference. Ann Int Med 93:141-144

68. Nemerson Y (1988) Tissue factor and haemostasis. Blood 71:1-8

69. Olson RE (1987) Vitamin K. In: Colman RW, Hirsh J, Marder VI, Salzman EW (eds) Hemostasis and thrombosis: basic principles and clinical practice. Lippincott, Philadelphia pp 846-860

70. Phillips LL (1984) Transfusion support in acquired coagulation disorders. Clin Haematol 13:137-150

71. Pickering NJ, Brody JI, Fink GB, Finnegan JO, Ablaza S (1983) The behaviour of antithrombin III, alpha2 macroglobulin, and alpha1 antitrypsin during cardiopulmonary bypass surgery. Am J Clin Pathol 80:459-464

72. Pineo GF, Gallus AS, Hirsh J (1973) Unexpected vitamin $\mathrm{K}$ deficiency in hospitalised patients. Can Med Assoc J 109:880-883

73. Rao AK, Pratt C, Berke A et al. (1988) Thrombolysis in Myocardial Infarction (TIMI) Trial - Phase I: Haemorrhagic manifestations and changes in plasma fibrinogen and the fibrinolytic system in patients treated with recombinant tissue plasminogen activator and streptokinase. J Am Coll Cardiol 11:1-11

74. Ratnoff OD (1984) Hemostatic defects in liver and biliary tract disease and disorders of vitamin $\mathrm{K}$ metabolism. In: Ratnoff OD, Forbes CD (eds) Disorders of hemostasis. Grune and Stratton, New York, pp 451-472

75. Remuzzi G (1988) Bleeding in renal failure. Lancet i: $1205-1208$

76. Remuzzi G, Benigni A, Dodesini P et al. (1982) Platelet function in patients on maintenance haemodialysis: depressed or enhanced? Clin Nephrol 17:60-63

77. Rickles FR, Edwards RL (1983) Activation of blood coagulation in cancer: Trousseau's sign revisited. Blood 62:14-20
78. Royston D, Bidstrup BP, Taylor KM, Sapsford RN (1987) Effect of Aprotinin on need for blood transfusion after repeat open-heart surgery. Lancet ii:1289-1291

79. Saldeen T (1983) Clotting, microembolism, and inhibition of fibrinolysis in adult respiratory distress. Surg Clin North Am 63:285-304

80. Salzman EW, Weinstein MJ, Weintraub RM et al. (1986) Treatment with desmopressin acetate to reduce blood loss after cardiac surgery. N Engl J Med 314:1402-1406

81. Schafer AI (1985) The hypercoagulable states. Ann Int Med 102:814-823

82. Sharp AA (1977) Diagnosis and management of disseminated intravascular coagulation. Br Med Bull 33:265-272

83. Sicard GA, Schier JJ, Totty WG et al. (1985) Thrombolytic therapy for acute arterial occlusion. J Vasc Surg 2:65-78

84. Stein SF, Harker LA (1982) Kinetic and functional studies of platelets, fibrinogen, and plasminogen in patients with hepatic cirrhosis. J Lab Clin Med 99:317230

85. Steiner RW, Coggins C, Carvalho AC (1979) Bleeding time in uremia: a useful test to assess clinical bleeding. Am J Haematol 7:107-117

86. Stibbe J, Kluft C, Brommer EJP, Gomes M, de Jong DS, Nauta J (1984) Enhanced fibrinolytic activity during cardiopulmonary bypass in open-heart surgery in man is caused by extrinsic (tissue-type) plasminogen activator. Eur J Clin Invest 14:375-382

87. Talbert IM, Blair PM (1979) Disseminated intravascular coagulation in obstetrics. Clin Obstet Gynaecol 22: $889-900$

88. Touho H, Hirakawa J, Hino A, Karasawa J, Ohno Y (1986) Relationship between abnormalities of coagulation and fibrinolysis and postoperative intracranial haemorrhage in head injury. Neurosurgery 19:523-531

89. Turney JH, Woods HF, Fewell MR, Weston MJ (1981) Factor VIII complex in uremia and effects of haemodialysis. Br Med J 282:1663

90. van der Sande JJ, Veltkamp JJ, Boekhout-Mussert RJ, Bouwhuis-Hoogerwerf ML (1978) Head injury and coagulation disorders. J Neurosurg 49:357-365

91. Weinstein M, Ware JA, Troll J, Salzman E (1988) Changes in von Willebrand factor during cardiac surgery: effect of desmopressin acetate. Blood 71 : 1648-1655

92. Weston MJ, Langley PG, Rubin MH, Hanid MA, Mellon P, Williams R (1977) Platelet function in fulminant hepatic failure and the effect of charcoal hemoperfusion. Gut 18:897-902

93. Whitaker AN, Elms MJ, Masci PP (1984) Measurement of cross linked fibrin derivatives in plasma: an immunoassay using monoclonal antibodies. J Clin Pathol 37: $882-890$

94. Zusman RM, Rubin RH, Cato AE, Cocchetto DM, Crow JW, Tolkoff-Rubin N (1981) Hemodialysis using prostacyclin instead of heparin as the sole anti-thrombotic agent. N Engl J Med 304:934-939 